\title{
Evidence-based algorithm for diagnosis and assessment in psoriatic arthritis: results by Italian DElphi in psoriatic Arthritis (IDEA)
}

\author{
G. Lapadula 1 , A. Marchesoni², F. Salaffi ${ }^{3}$, R. Ramonda ${ }^{4}$, C. Salvarani ${ }^{5}$, L. Punzi ${ }^{6}$, \\ L. Costa ${ }^{7}$, F. Caso ${ }^{6}$, D. Simone ${ }^{8}$, G. Baiocchi ${ }^{9}$, C. Scioscia ${ }^{10}$, M. Di Carlo ${ }^{11}$, \\ R. Scarpa ${ }^{7}$, G. Ferraccioli ${ }^{8}$ \\ ${ }^{1}$ Dipartimento Interdisciplinare di Medicina, Università degli Studi di Bari Aldo Moro; ${ }^{2}$ Dipartimento di \\ Reumatologia, Istituto Ortopedico Gaetano Pini, Milano; ${ }^{3}$ Dipartimento di Reumatologia, Università \\ Politecnica delle Marche, Ancona; ${ }^{4}$ Dipartimento di Reumatologia, Università degli Studi di Padova; \\ ${ }^{5}$ Dipartimento di Chirurgia, Università degli Studi di Modena e Reggio Emilia; ${ }^{6}$ Dipartimento di Medicina, \\ Università degli Studi di Padova; 'Dipartimento di Medicina Clinica e Chirurgia, Università degli Studi di Napoli \\ Federico II; ${ }^{8}$ Dipartimento di Reumatologia, Università Cattolica del Sacro Cuore, Milano; ' Unità Operativa \\ di Reumatologia, Arcispedale S. Maria Nuova, Reggio Emilia; ${ }^{10}$ Unità Operativa di Reumatologia, Università \\ degli Studi di Bari Aldo Moro; ${ }^{11}$ Dipartimento di Reumatologia, Presidio Ospedali Riuniti Murri-Jesi, AN
}

\section{SUMMARY}

Psoriatic arthritis (PsA) is a chronic inflammatory disease involving skin, peripheral joints, entheses, and axial skeleton. The disease is frequently associated with extrarticular manifestations (EAMs) and comorbidities. In order to create a protocol for PsA diagnosis and global assessment of patients with an algorithm based on anamnestic, clinical, laboratory and imaging procedures, we established a DElphi study on a national scale, named Italian DElphi in psoriatic Arthritis (IDEA).

After a literature search, a Delphi poll, involving 52 rheumatologists, was performed. On the basis of the literature search, 202 potential items were identified.

The steering committee planned at least two Delphi rounds. In the first Delphi round, the experts judged each of the 202 items using a score ranging from 1 to 9 based on its increasing clinical relevance. The questions posed to experts were How relevant is this procedure/observation/sign/symptom for assessment of a psoriatic arthritis patient? Proposals of additional items, not included in the questionnaire, were also encouraged. The results of the poll were discussed by the Steering Committee, which evaluated the necessity for removing selected procedures or adding additional ones, according to criteria of clinical appropriateness and sustainability.

A total of 43 recommended diagnosis and assessment procedures, recognized as items, were derived by combination of the Delphi survey and two National Expert Meetings, and grouped in different areas. Favourable opinion was reached in $100 \%$ of cases for several aspects covering the following areas: medical (familial and personal) history, physical evaluation, imaging tool, second level laboratory tests, disease activity measurement and extrarticular manifestations. After performing PsA diagnosis, identification of specific disease activity scores and clinimetric approaches were suggested for assessing the different clinical subsets.

Further, results showed the need for investigation on the presence of several EAMs and risk factors. In the context of any area, a rank was assigned for each item by Expert Committee members, in order to create the logical sequence of the algorithm. The final list of recommended diagnosis and assessment procedures, by the Delphi survey and the two National Expert Meetings, was also reported as an algorithm.

This study shows results obtained by the combination of a DElphi survey of a group of Italian rheumatologists and two National Expert Meetings, created with the aim of establishing a clinical procedure and algorithm for the diagnosis and the assessment of PsA patients.

In order to find accurate and practical diagnostic and assessment items in clinical practice, we have focused our attention on evaluating the different PsA domains. Hence, we conceived the IDEA algorithm in order to address PsA diagnosis and assessment in the context of daily clinical practice.

The IDEA algorithm might eventually lead to a multidimensional approach and could represent a useful and practical tool for addressing diagnosis and for assessing the disease appropriately.

Corresponding author

However, the elaborated algorithm needs to be further investigated in daily practice, for evidencing and proving its eventual efficacy in detecting and staging PsA and its heterogeneous spectrum appropriately.

Key words: Rheumatology; arthritis; psoriatic arthritis; psoriasis; comorbidities; diagnosis algorithm. 


\section{INTRODUCTION}

Dsoriatic arthritis (PsA) is a chronic inflammatory disease involving skin, peripheral joints, entheses, and axial skeleton. The disease is frequently associated with extrarticular manifestations (EAMs) and comorbidities (1). Diagnosis relies mainly on clinical evaluation and the CASPAR (ClASsification criteria for Psoriatic ARthritis) criteria are often used in epidemiological and research studies, having high specificity $(98.7 \%)$ and sensitivity $(91.4 \%)(2,3)$.

Frequently, peripheral patterns overlap with axial involvement leading to different phenotypical combinations and frequency, in which dactylitis, enthesitis, low back pain, oligo and mono arthritis significantly characterize the disease (4-7). Cutaneous domain represents another aspect leading to wide phenotypical heterogeneity. Psoriasis generally occurs before articular manifestations; it can be contemporaneous with, or later than arthritis. Furthermore, the case of patients with arthritis and familiar psoriasis provides a PsA subset classified as sine psoriasis (8).

In addition, in recent years the increase of clinical studies have outlined how PsA can be associated with extrarticular manifestations, also alternatively recognized as comorbities (9-13). Among these, the most frequent are represented by uveitis (9), colitis (10), metabolic syndrome (MS) (11) and involvement of the cardiovascular system (12). Psychological aspects, such as depressive symptoms and anxiety, represent important correlates of health related quality of life (HRQoL) (13).

Hence, the new concept of psoriatic disease has identified this heterogeneous condition, abandoning the view of PsA as a merely articular and cutaneous mild inflammatory state (14).

However, once the patient has been diagnosed with PsA, the variable clinical spectrum can make the assessment of disease activity a challenge both for articular and cutaneous aspects and for systemic manifestations $(15,16)$.

In order to find accurate, reliable, and fea- sible activity measures useful in longitudinal cohorts, clinical trials, and clinical practice, the Group for Outcome Measures in Rheumatology (OMERACT), and for Research and Assessment of Psoriasis and Psoriatic Arthritis (GRAPPA), have focused their attention on refining and assessing the different PsA domains (16).

In particular, to assist the rheumatologist in the management of PsA, OMERACT Group proposed a core set of six core domains, represented by peripheral joint and skin activity, pain, patient global assessment (PGA), physical function, and HRQoL. In addition, spinal disease, dactylitis, enthesitis, fatigue, nail disease, radiography, and acute-phase indices were considered important domains (17).

GRAPPA group highlights that assessment of PsA patients requires full consideration of all major disease domains, including peripheral arthritis, axial disease, enthesitis, dactylitis, psoriasis, and nail disease. Further, the impact of PsA on pain, function, QoL, and structural damage needs to be assessed (18). In addition, a comprehensive assessment of other potential related conditions should be considered, including uveitis, inflammatory bowel disease (IBD), cardiovascular disease, obesity, MS, gout, diabetes mellitus (DM), liver disease, depressive and anxiety-symptoms (18-22).

In recent years, in psoriasis (23) and PsA (24-29), several studies have applied Delphi procedures $(30,31)$ for different aims, mainly focused on better diagnosis and assessment of these clinical conditions.

In particular, in 2015, in the context of psoriasis, Italian dermatologists performed a Delphi procedure involving 50 dedicated dermatological centres (23). This led to the definition of a multidimensional assessment algorithm for psoriasis diagnosis and assessment which was potentially useful for proving sensitivity sustainable in daily clinical practice, named PSOCUBE. It includes a three-dimensional table comprising 14 clinical examinations and historyrecording items, 32 laboratory screenings and instrumental exams and 11 clinimetric scores (23).

In order to create PsA diagnosis and global 
assessment protocol and an algorithm based on anamnestic, clinical, laboratory and imaging procedures, we established a Delphi study on a national scale, named Italian DElphi in psoriatic Arthritis (IDEA).

\section{MATERIALS AND METHODS}

As a first step, all the evaluations (patient anamnesis, clinical evaluation, laboratory tests, imaging procedures) considered useful for the diagnosis, and the domains for the assessment, were identified by an expert board dictating the items, through a literature search.

Subsequently, a Delphi poll involving 52 rheumatologists, representative of the Italian rheumatologic community and with expertise in current good clinical practice for PsA, was performed. We used a modified Delphi technique in which questionnaire rounds were followed by a well-structured meeting of the Steering Committee, to discuss and validate (by voting) the results.-

\section{Delphi questionnaire preparation}

Articles published in indexed English language journals on randomized controlled clinical trials, meta-analysis, guidelines, reviews and observational studies dealing specifically with psoriatic arthritis and its comorbidities, were selected by the authors. The articles were identified by a MEDLINE, EMBASE, Cochrane Library, PubMed using the keywords psoriatic arthritis and/or psoriasis, matched with several keywords, relevant for every aspect of the diseases, including also the term comorbidities.

On the basis of the literature search, we identified a preliminary list of 202 potential items useful for assessment of PsA, subdivided in key domains necessary to assess and possibly to distinguish PsA from other rheumatic diseases. In particular the key domains were: musculoskeletal, dermatological, metabolic, cardiologic, psychiatric and quality of life related, gastroenterological, and ophthalmologic.

\section{The survey}

the steering committee planned at least two Delphi rounds. The consensus process was conducted via email. In the first Delphi round the experts judged each of the 202 items using a score ranging from 1 to 9 based on its increasing clinical relevance. The questions posed to experts was How relevant is this procedure/observation/ sign/symptom for assessment of a psoriatic arthritis patient? Proposals of additional items, not included in the questionnaire, were also encouraged. Crucial to the final results were: speed of transmission, maintenance of respondent anonymity, and potential for rapid feedback.

The agreement was defined when a score was reached by at least $80 \%$ of the experts. The criteria for agreement and disagreement between experts were defined as follows:

1) agreement - when $80 \%$ of the panellists' ratings fall into one of the 3-bands in a scale from 1 to 9 (1-3; 4-6; 7-9);

2) disagreement - when $90 \%$ of the panellists' ratings fall instead into one of two extra-wide bands (1-6 or 4-9).

It means that if, to reach the $90 \%$ votes, we need to include two large intervals, the distribution is too skewed and not symmetrical. As such, it represents disagreement.

In the second round, participants were asked to rate again only the procedure that did not reach the optimal levels of agreement and disagreement.

The results of the poll were discussed by the Steering Committee, which evaluated the necessity for removing selected procedures or adding additional ones, according to criteria of clinical appropriateness and sustainability.

The final list, made of 43 items grouped in the previously agreed key domains, was discussed in a first 1-day National Expert Meeting in October 2014, involving 30 participants. Further, in this meeting it was decided to use the items to prepare an algorithm to assess the patient in daily clinical practice. In light of this, an additional round of the Delphi exercise was prepared to select through a priority level (high, medium, low) those items considered worthy of inclusion in the different steps of the flow-chart (laboratory data, history, physical exam, etc). The main steps of the study are reported in Figure 1. 


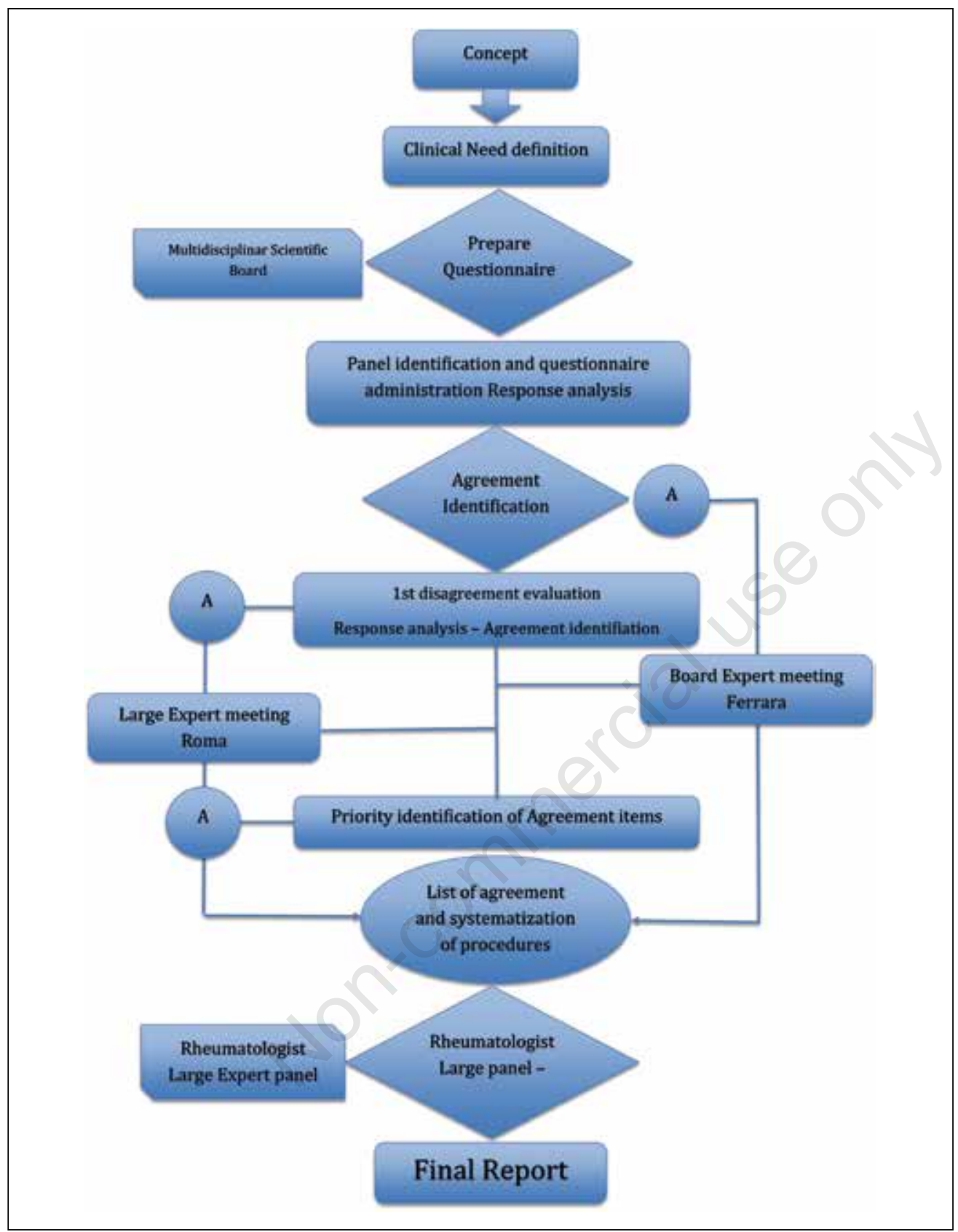

Figure 1 - Main steps of the Italian Delphi in Psoriatic Arthritis (IDEA) project.

Calculations were performed using the Office 2007 software package.

\section{RESULTS}

A total of 43 recommended diagnosis and assessment procedures, recognized as items, were derived by a combination of the Delphi survey and two National Expert
Meetings, and grouped in different areas including medical (familial and personal) history, physical evaluation, imaging tool, second level laboratory tests, disease activity measurement and extrarticular manifestations (Table I). For each of these, the frequency of high, middle and low priorities are also reported.

A first level laboratory tests area was also 
Table I - The 43 recommended diagnostic and assessment procedures, recognized as items, derived by combination of the DElphi survey and two National Expert Meetings, and grouped in different areas including medical (familial and personal) history, physical evaluation, imaging tool, second level laboratory tests, disease activity measurement and extrarticular manifestations.

\begin{tabular}{|c|c|}
\hline Area & Item \\
\hline $1^{\text {st }}$ level laboratory tests & Cellular blood count (CBC) \\
\hline $1^{\text {st }}$ level laboratory tests & Creatinine \\
\hline $1^{\text {st }}$ level laboratory tests & ALT/GPT \\
\hline $1^{\text {st }}$ level laboratory tests & ESR \\
\hline $1^{\text {st }}$ level laboratory tests & CRP \\
\hline $1^{\text {st }}$ level laboratory tests & Urinalysis \\
\hline Medical history & Diagnosis of psoriasis and/or psoriatic onychopathy \\
\hline Medical history & Psoriasis in 1st and 2nd degree relatives \\
\hline Medical history & $\begin{array}{l}\text { Site and symptoms characteristics (pain and/or swelling } \\
\text { and/or stiffness) }\end{array}$ \\
\hline Medical history & Symptoms duration \\
\hline Medical history & Arthritis and/or spondylitis familiarity \\
\hline Family medical history & Personal or familiar hystory of inflammatory bowel disease \\
\hline Physical evaluation & Site and number of swollen joints \\
\hline Physical evaluation & Site and number of tender joints \\
\hline Physical evaluation & Insertional pain in enthesitis sites \\
\hline Physical evaluation & $\begin{array}{l}\text { In case of axial disease pattern, clinimetric evaluation of the spine } \\
\text { (tragus-to-wall distance, lateral bending, Schober's test, cervical } \\
\text { spine rotation, inter-malleolar distance) }\end{array}$ \\
\hline Physical evaluation & Presence of psoriasis and/or onychopathy \\
\hline Imaging & X-ray of the involved joints \\
\hline Imaging & US of the involved joints and enthesis \\
\hline Imaging & MRI of the sacroiliac joints \\
\hline 2nd level laboratory tests & CRP \\
\hline 2nd level laboratory tests & HLA-B27 (only if axial and/or enthesis symptoms) \\
\hline 2nd level laboratory tests & ACPA + Rheumatoid factor \\
\hline 2nd level laboratory tests & Uricaemia \\
\hline Involved sites & Pattern of localization of disease symptoms \\
\hline Disease activity measurement & Number of tender joints (TJ 68) and swollen joints (SJ 66) \\
\hline Disease activity measurement & LEI $(0-6)$ \\
\hline Disease activity measurement & Pain VAS $(0-10)$ \\
\hline Disease activity measurement & BASDAI \\
\hline Disease activity measurement & BASFI \\
\hline Disease activity measurement & PGA $(0-10)$ \\
\hline Disease activity measurement & $\mathrm{HAQ}$ \\
\hline Comorbidities & $\begin{array}{l}\text { Cardiovascular events, hypertension, diabetes, obesity, dyslipidemia } \\
\text { ed hyperuricemia }\end{array}$ \\
\hline Comorbidities & General visit (heart, lung, abdomen, skin and annexes) \\
\hline Comorbidities & $\begin{array}{l}\text { Sending the specialist areas of expertise must be selective and } \\
\text { based on patient characteristics }\end{array}$ \\
\hline Ocular comorbidity & Active uveitis and relapses number \\
\hline
\end{tabular}




\begin{tabular}{|l|l|}
\hline Physical evaluation & Dactylitis (sites and numbers) \\
\hline Gastroenteric comorbidity & Personal or family history of IBD \\
\hline Dermatologic comorbidity & PASI \\
\hline Psycological comorbidity \& QoL & Presence/absence fybromyalgia \\
\hline Psycological comorbidity \& QoL & Performing of PsAID-12 test is recommended \\
\hline Dismetabolic comorbidity & Metabolic syndrome \\
\hline Dismetabolic comorbidity & Obesity \\
\hline
\end{tabular}

ALT/GPT, alanine serum transaminases; ESR, erythrocyte sedimentation rate; CRP, C-reactive protein; US, ultrasound; MRI, magnetic resonance imaginf; ACPA, anti-citrullinated protein antibodies; LEl, Leeds enthesitis index; VAS, visual analogic scale pain; BASDAl, bath ankylosing spondylitis disease activity index; BASFI, bath ankylosing spondylitis functional index; PGA, patient global assessment; HAQ, health assessment questionnaire; IBD, inflammatory bowel disease; PASI, psoriasis area severity index; PsAID-12, psoriatic arthritis impact of disease questionnaire; QoL, quality of life.

added by expert committee members as described successively.

\section{Anamnestic data}

In the anamnestic phase, for each patient suspected of PsA, useful investigations were represented by diagnosis of psoriasis and/or psoriatic onychopathy in personal history (favorable opinion in $100 \%$ of cases with high priority in $100 \%$ ) and of psoriasis in $1^{\text {st }}$ and $2^{\text {nd }}$ degree relatives (favorable opinion in $100 \%$ of cases with high and middle priority respectively, in $92.6 \%$ and $7.4 \%$ ).

Other important anamnestic aspects for PsA diagnosis were considered familial history for arthritis and spondylitis (favorable opinion in $96 \%$ of cases with high, middle and low priority respectively in $37 \%, 51.9 \%$ and $11.1 \%$ ), site and pattern of articular involvement (pain, swelling and stiffness) (favorable opinion in $89 \%$ of cases with high and middle priority respectively, in $88.9 \%$ and $11.1 \%$ ) and disease duration (favorable opinion in $100 \%$ of cases with high, middle and low priority respectively, in $59.3 \%, 33.3$ and $7.4 \%$ ).

\section{Physical evaluation}

In the context of physical examination, favourable opinion was reached in 100\% of cases for the following items: swollen joints count (SJC) (high and middle priority respectively in $85.2 \%$ and $14.8 \%$ ); tender joints count (TJC) (high, middle and low priority respectively in $85.2 \%, 11.1 \%$ and 3.7\%); tender entheseal sites (high and middle priority respectively in $88.9 \%$ and $11.1 \%$ ); dactylitis (sites and numbers) (high and middle priority respectively in $96.3 \%$ and $3.7 \%$ ); clinimetric evaluation of the spine (tragus-to-wall distance, lateral bending, Schober's test, cervical spine rotation, inter-malleolar distance) (high, middle and low priority respectively in $63 \%, 33.3 \%$ and $3.7 \%$ ); presence of psoriasis and/or onychopathy (high, middle and low priority respectively in $92.6 \%, 3.7 \%$ and $3.7 \%$ ).

\section{Imaging tools}

Favorable opinion was reached in 100\% of cases for the following imaging items: Xray of the involved joints (high and middle priority respectively, in $80 \%$ and $20 \%$ ); US of the involved joints and enthesis (high and middle priority respectively in $74.1 \%$ and $25.9 \%$ ) (high and middle priority respectively in $85.2 \%$ and $14.8 \%$ ); magnetic resonance imaging (MRI) of the sacroiliac joints in presence of inflammatory back pain (high and middle priority respectively in $88.9 \%$ and $11.1 \%$ ).

\section{Second level laboratory tests}

In the context of laboratory evaluation, favourable opinion was reached in $100 \%$ of cases for the following items: HLA-B27 in patients with axial and entheseal involvement (high, middle and low priority respectively in $44.4 \%, 51.9 \%$ and $3.7 \%$ ); C-RP (high, and middle priority respectively in $73.1 \%$, and $26.9 \%$ ); uricaemia (high, mid- 
dle and low priority respectively in $29.6 \%$, $55.6 \%$ and $14.8 \%$ ).

It was suggested that anti-citrullinated protein antibodies (ACPA) and rheumatoid factor (RF) should be evaluated in case of differential diagnosis with rheumatoid arthritis (RA) (high, middle and low priority respectively in $37 \%, 48.1 \%$ and $14.8 \%$ ).

\section{Clinimetric approach}

Favourable opinion was reached in $100 \%$ of cases for the following clinimetric items: 68-TJC and 66-SJC (for both, high and middle priority respectively in $92.6 \%$ and $7.4 \%$ of cases); Bath ankylosing spondylitis disease activity index (BASDAI) (high and middle priority respectively in $81.5 \%$ and 18.5\%); Bath ankylosing spondylitis functional index (BASFI), health assessment questionnaire (HAQ) and visual analogic scale (VAS)-pain (for all, high and middle priority respectively in $63 \%$ and 37\%); Leeds enthesitis index (LEI) (0-6) (high, middle and low priority respectively in $37 \%, 51 \%$ and $11.1 \%$ ); PGA (0-10) (high, middle and low priority respectively in $55.6 \%, 40.7 \%$, and $3.7 \%$ of cases).

\section{Psoriatic arthritis classification}

After performing PsA diagnosis, identification of clinical subsets, specific disease activity scores and clinimetric approach were suggested for assessing the disease.

Among these, the most important were considered to be BASDAI, BASFI, HAQ, VAS and PGA in axial pattern; 68-TJC, 66-SJC, HAQ, VAS, PGA in peripheral pattern; LEI (0-6), HAQ, VAS, PGA in enthesitis; HAQ, VAS, and PGA in dactylitis.

\section{Extrarticular manifestations}

After performing PsA diagnosis, identification of associated extrarticular manifestations was considered of relevance as result of the study. In particular, the results showed the need for investigation on the presence of several extrarticular manifestations and risk factors.

In particular, in the anamnestic phase, the results evidenced the need to focus on cardiovascular events, systolic and/or diastolic hypertension, DM, obesity, dyslipidaemia and hyperuricemia (favorable opinion in $100 \%$ of cases with high and middle priority in $74.1 \%$ and $25.9 \%$ ).

The presence of high psoriasis area severity index (PASI), MS, obesity, uveitis or recurrence of uveitis, personal and/or familial history of IBD, psychological comorbidity and low QoL tested by the psoriatic arthritis impact of disease questionnaire, represented important tools for addressing the patient towards the specialist area of expertise (favorable opinion in $100 \%$ of cases with high, middle and low priority respectively in $55.6 \%, 37 \%$, and $7.4 \%$ ).

At the first patient interview, expert committee members recommended performing first level laboratory tests such as cellular blood count (CBC), serum creatinine, aspartate and alanine serum transaminases (AST and ALT), erythrocyte sedimentation rate (ESR), and urinalysis. These were included in the first level laboratory tests area.

In the context of each area, a rank was assigned for each item by Expert Committee members, in order to create the logical sequence for the algorithm.

The final list of recommended diagnosis and assessment procedures, by the Delphi survey and the two National Expert Meetings, is shown in Table I and as an algorithm in Figure 2.

\section{DISCUSSION}

This study shows results obtained by the combination of a Delphi survey of a group of Italian rheumatologists and two National Expert Meetings, created with the aim of establishing a clinical procedure and algorithm for the diagnosis and the assessment of PsA patients.

In PsA, due to the absence of recent diagnostic criteria and of well-defined indices assessing the heterogeneity of the condition, both diagnosis and assessment need of be deeply investigated. Reduction of PsA severity, improvement of QoL and psychosocial components are mainly related to early diagnosis and appropriate assessment with prompt therapeutic intervention (32-34). 


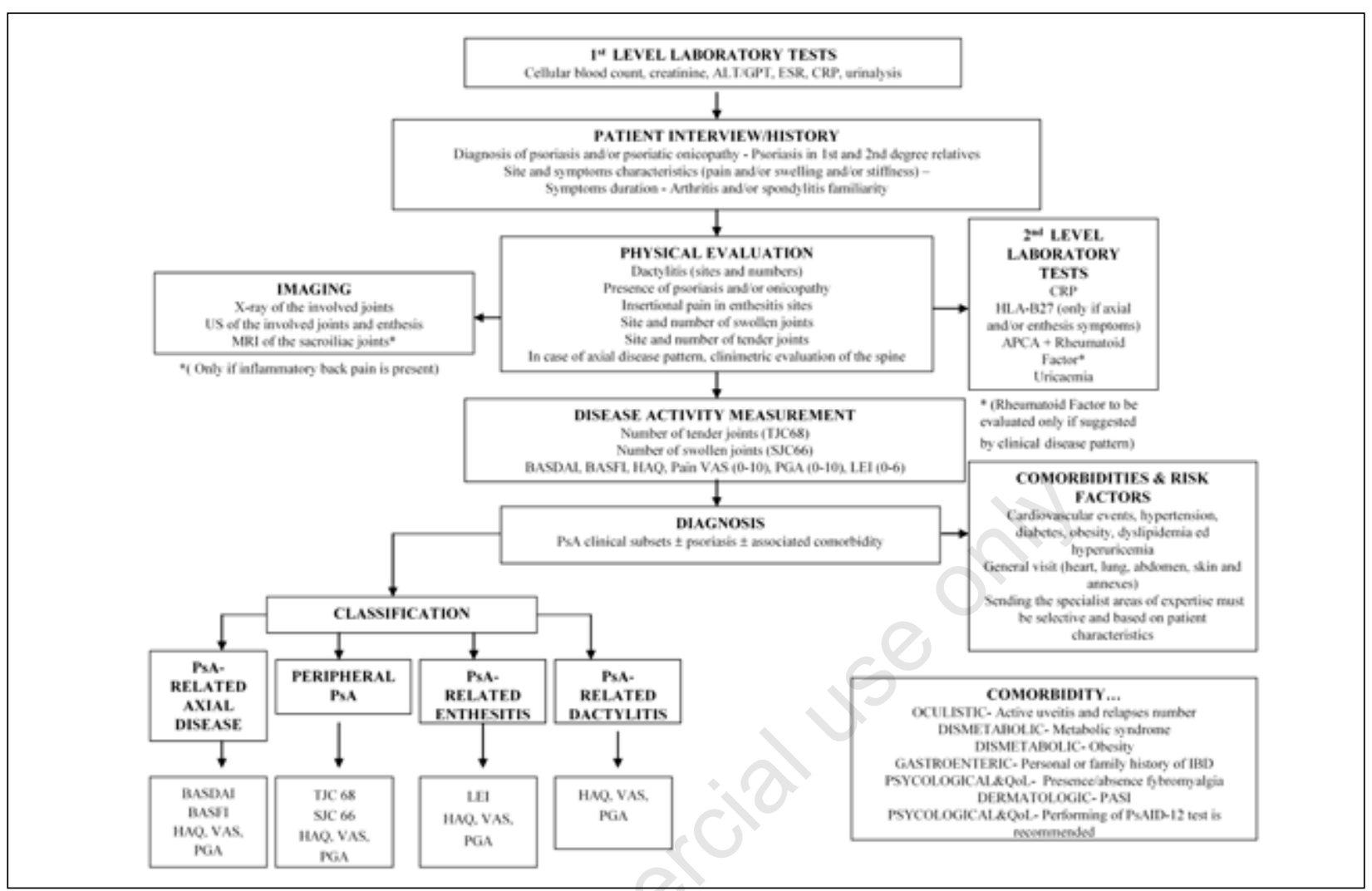

Figure 2 - Italian DElphi in Psoriatic Arthritis (IDEA) algorithm reporting the final list of recommended diagnosis and assessment procedures, by the Delphi survey and the two National Expert Meetings.

The final goal in PsA therapy is to inhibit structural radiological damage, induce clinical remission, and improve patients' QoL, as defined by GRAPPA and the European League Against Rheumatism in the European League Against Rheumatism (EULAR) recommendations (19, 35-38).

Hence, we have hypothesised a Delphi study for establishing a consensus from a large group of rheumatologists with the aim of improving PsA early diagnosis and assessing the disease and its heterogeneous aspects.

Forty-three recommended items reaching high frequency of favourable opinion in this study included both diagnostic and assessment procedures. Items were then grouped in different areas covering medical (familial and personal) history, physical evaluation, imaging, second level laboratory tests, disease activity measurement and extrarticular manifestations (ocular, gastrointestinal, dermatological, psychological, and dismetabolic manifestations). A first level laboratory tests area was also added by expert committee members.

In particular, the results of the study showed that diagnosis of psoriasis and/or psoriatic onychopathy in personal history, psoriasis in $1^{\text {st }}$ and $2^{\text {nd }}$ degree relatives, familiar history for arthritis and spondylitis, articular site and pattern, and disease duration represented the most important anamnestic items. In the context of physical evaluation, the most important items were represented by dactylitis (sites and numbers), presence of psoriasis and/or onychopathy, tender entheseal sites, SJC and TJC, clinimetric evaluation of the spine (tragus-towall distance, lateral bending, Schober's test, cervical spine rotation, inter-malleolar distance). Articular X-rays, US of the involved joints and enthesis and MRIs of the sacroiliac joints in presence of inflammatory back pain provided the most important imaging findings. 
The following key laboratory findings were suggested: C-RP, HLA-B27 in patients with axial and entheseal involvement, and uricaemia. ACPA and RF were considered important in the differential diagnosis with RA. Further, first level laboratory tests such as CBC, serum creatinine, AST and ALT, ESR, and urinalysis were recommended by expert committee members, to be performed at the first patient interview.

With regard to the clinimetric approach, the most important items were represented by 68 -TJC and 66-SJC, BASDAI, BASFI, HAQ, VAS-pain, LEI (0-6), and PGA (0$10)$.

In addition, expert committee members recommended the calculation of the DAPSA score (39). Disease activity index for psoriatic arthritis (DAPSA) is a diseasespecific validated and feasible tool for PsA assessment. Recently, DAPSA has been considered as reference tool for defining remission or low disease activity within the first recommendation of the latest EULAR recommendations on PsA (38).

Anamnestic investigation on the presence of cardiovascular events, systolic and/or diastolic hypertension, DM, obesity, and dyslipidemia were suggested as important findings for the assessment of the disease. Among extrarticular manifestations, the presence of high PASI, MS, obesity, uveitis or recurrent uveitis, personal and/or familial history of IBD, and psychological aspects needed to be evaluated.

In order to find accurate and practical diagnostic and assessment items in clinical practice, we have focused our attention on evaluating the different PsA domains. Hence, we conceived the IDEA algorithm in order to address PsA diagnosis and assessment in the context of daily clinical practice.

In accordance with OMERACT (17) and GRAPPA groups (18-22), this study revealed the need to assess PsA with full consideration of all major disease domains, including peripheral arthritis, axial disease, enthesitis, dactylitis, and psoriasis, both in anamnestic and clinical evaluation.

In particular, in this study, the presence of arthritis, dactylitis, enthesitis and axial in- volvement in the context of psoriasis and/ or its familiarity are confirmed as the main clinical items useful in addressing the diagnosis. Peculiar MRI and US imaging findings of involved articular and entheseal districts, evaluation of C-RP and HLAB27 positivity in case of axial involvement emerged as important elements for addressing the diagnosis and assessing the disease. RF and ACPA seronegativity represent useful findings for excluding RA. Further, in accordance with GRAPPA recommendations (18-22), this study confirms that rheumatologists should be also aware of differing extrarticular conditions, which require expert consultation to guarantee prompt global assessment.

\section{CONCLUSIONS}

In conclusion, the IDEA algorithm could represent a useful and practical tool for addressing the diagnosis and for assessing appropriately the disease. In addition, this algorithm might eventually lead to a multidimensional approach in which rheumatologists have to consider not only articular and cutaneous aspects, but also systemic aspects.

However, the elaborated algorithm needs to be further investigated in daily practice, for evidencing and for proving its eventual efficacy in detecting and staging appropriately PsA and its heterogeneous spectrum.

Contributors: Amato G., Anelli M.G., Batticciotto A., Benucci M., Bortolotti., Bruschi., Cantatore F.P., Carletto., Cassarà E., Casu C., Cauli A., Ciancio G., Ciapetti A., Cino N., D'onofrio F., De Marco G., Del Puente A., Desiati F., Erre G., Ferri C., Filippini M., Fusaro E., Giannitti C., Gibertini P., Gorla R., Govoni M., Gremese E., Guggino G., Gutierrez M., Li Gobbi F., Manganelli S., Mozzani F., Niccoli L., Nowik M., Ostuni P., Paolazzi G., Pellerito R., Peroni Clara L., Piga M., Pipitone N., Possemato N., Santilli D., Schavon F., Schinocca C., Sciortino D., Sebastiani M., Serafino L., Sfriso P., Spanò A., Ventura D., Visalli E., Vitetta R., Maruotti N. 


\section{REFERENCES}

1. Wright V, Moll JM. Psoriatic arthritis. Bull Rheum Dis. 1971; 21: 627-32.

2. Taylor W, Gladman D, Helliwell P, et al. Classification criteria for psoriatic arthritis: development of new criteria from a large international study. Arthritis Rheum. 2006; 54: 2665-73.

3. Tillett W, Costa L, Jadon D, et al. The ClASsification for Psoriatic ARthritis (CASPAR) criteria - a retrospective feasibility, sensitivity, and specificity study. J Rheumatol. 2012; 39: 154-6.

4. Scarpa R, Oriente P, Pucino A, et al. The clinical spectrum of psoriatic spondylitis. $\mathrm{Br}$ J Rheumatol. 1988; 27: 133-7.

5. Jones SM, Armas JB, Cohen MG, et al. Psoriatic arthritis: outcome of disease subsets and relationship of joint disease to nail and skin disease. Br J Rheumatol. 1994; 33: 834-9.

6. Caso F, Costa L, Atteno M, et al. Simple clinical indicators for early psoriatic arthritis detection. Springerplus. 2014; 3: 759.

7. Marchesoni A, Atzeni F, Spadaro A, et al. Identification of the clinical features distinguishing psoriatic arthritis and fibromyalgia. $\mathbf{J}$ Rheumatol. 2012; 39: 849-55.

8. Myers WA, Gottlieb AB, Mease P. Psoriasis and psoriatic arthritis: clinical features and disease mechanisms. Clin Dermatol. 2006; 24: 438-47.

9. Egeberg A, Khalid U, Gislason GH, et al. Association of psoriatic disease with uveitis: a danish nationwide cohort study. JAMA Dermatol. 2015; 151: 1200-5.

10. Scarpa R, Manguso F, D'Arienzo A, et al. Microscopic inflammatory changes in colon of patients with both active psoriasis and psoriatic arthritis without bowel symptoms. J Rheumatol. 2000; 27: 1241-6.

11. Costa L, Caso F, Ramonda R, et al. Metabolic syndrome and its relationship with the achievement of minimal disease activity state in psoriatic arthritis patients: an observational study. Immunol Res. 2015; 61: 147-53.

12. Costa L, Caso F, D'Elia L, et al. Psoriatic arthritis is associated with increased arterial stiffness in the absence of known cardiovascular risk factors: a case control study. Clin Rheumatol. 2012; 31: 711-5.

13. Kotsis K, Voulgari PV, Tsifetaki N, et al. Anxiety and depressive symptoms and illness perceptions in psoriatic arthritis and associations with physical health-related quality of life. Arthritis Care Res (Hoboken). 2012; 64: 1593-601.

14. Scarpa R, Ayala F, Caporaso N, Olivieri I. Psoriasis, psoriatic arthritis, or psoriatic disease? J Rheumatol 2006; 33: 210-2.

15. Scarpa R, Caso F, Costa L, et al. Psoriatic disease: clinical staging. J Rheumatol Suppl. 2015; 93: 24-6.
16. Coates LC, FitzGerald O, Mease PJ, et al. Development of a disease activity and responder index for psoriatic arthritis--report of the Psoriatic Arthritis Module at OMERACT 11. J Rheumatol. 2014; 41: 782-91.

17. Gladman DD, Mease PJ, Strand V, et al. Consensus on a core set of domains for psoriatic arthritis. J Rheumatol. 2007; 34: 1167-70.

18. Boehncke WH. GRAPPA 2013 basic/translational/clinical science update: comorbidity monitoring. J Rheumatol. 2014; 41: 1224-6.

19. Coates LC, Kavanaugh A, Mease PJ, et al. Group for Research and Assessment of Psoriasis and Psoriatic Arthritis 2015 Treatment Recommendations for Psoriatic Arthritis. Arthritis Rheumatol. 2016 [Epub ahead of print].

20. Ogdie A, Schwartzman S, Eder L, et al. Comprehensive treatment of psoriatic arthritis: managing comorbidities and extraarticular manifestations. J Rheumatol. 2014; 41: 231522.

21. Armstrong AW, Gelfand JM, Boehncke WH, Armstrong EJ. Cardiovascular comorbidities of psoriasis and psoriatic arthritis: a report from the GRAPPA 2012 annual meeting. J Rheumatol. 2013; 40: 1434-7.

22. Raychaudhuri SP. Comorbidities of psoriatic arthritis - metabolic syndrome and prevention: a report from the GRAPPA 2010 annual meeting. J Rheumatol. 2012; 39: 437-40.

23. Linder D, Altomare G, Amato S, et al. PSOCU$\mathrm{BE}$, a multidimensional assessment of psoriasis patients as a both clinically/practically sustainable and evidence-based algorithm. J Eur Acad Dermatol. Venereol. 2015; 29: 1310-7.

24. Roubille C, Richer V, Starnino T, et al. Evidence-based recommendations for the management of comorbidities in rheumatoid arthritis, psoriasis, and psoriatic arthritis: expert opinion of the Canadian Dermatology-Rheumatology Comorbidity Initiative. J Rheumatol. 2015; 42: 1767-80.

25. Boehncke WH, Anliker MD, Conrad C, et al. The dermatologists' role in managing psoriatic arthritis: results of a Swiss DElphi exercise intended to improve collaboration with rheumatologists. Dermatology. 2015; 230: 75-81.

26. Cañete JD, Daudén E, Queiro R, et al. Recommendations for the coordinated management of psoriatic arthritis by rheumatologists and dermatologists: a DElphi study. Actas Dermosifiliogr. 2014; 105: 216-32.

27. Maksymowych WP, Landewé R, Tak PP, et al. Reappraisal of OMERACT 8 draft validation criteria for a soluble biomarker reflecting structural damage endpoints in rheumatoid arthritis, psoriatic arthritis, and spondyloarthritis: the OMERACT 9 v2 criteria. J Rheumatol. 2009; 36: 1785-91.

28. Lavie F, Salliot C, Dernis E, et al. Prognosis and follow-up of psoriatic arthritis with peripheral joint involvement: development of 
recommendations for clinical practice based on published evidence and expert opinion. Joint Bone Spine. 2009; 76: 540-6.

29. Taylor WJ. Preliminary identification of core domains for outcome studies in psoriatic arthritis using DElphi methods. Ann Rheum Dis. 2005; 64: ii110-2.

30. Dalkey NC. Delphi. Santa Monica, CA: Rand; 1967.

31. Normand SL, McNeil BJ, Peterson LE, et al. Eliciting expert opinion using the DElphi technique: identifying performance indicators for cardiovascular disease. Int J Qual Health Care. 1998; 10: 247-60.

32. McHugh NJ. Early psoriatic arthritis. Rheum Dis Clin North Am. 2015; 41: 615-22.

33. Caso F, Lubrano E, Del Puente A, et al. Progress in understanding and utilizing TNF- $\alpha$ inhibition for the treatment of psoriatic arthritis. Exp Rev Clin Immunol. 2016; 12: 315-31.

34. Caso F, Del Puente A, Peluso R, et al. Emerging drugs for psoriatic arthritis. Exp Opin Emerg Drugs. 2016; 21: 69-79.

35. Ritchlin CT, Kavanaugh A, Gladman DD, et al. Group for Research and Assessment of
Psoriasis and Psoriatic Arthritis (GRAPPA). Treatment recommendations for psoriatic arthritis. Ann Rheum Dis. 2009; 68: 1387-94.

36. Coates LC, Ritchlin CT, Kavanaugh AF. GRAPPA treatment recommendations: an update from the GRAPPA 2013 Annual Meeting. J Rheumatol. 2014; 41: 1237-9.

37. Ash Z, Gaujoux-Viala C, Gossec L, et al. A systematic literature review of drug therapies for the treatment of psoriatic arthritis: current evidence and meta-analysis informing the EULAR recommendations for the management of psoriatic arthritis. Ann Rheum Dis. 2012; 71: 319-26.

38. Gossec L, Smolen JS, Ramiro S, et al. European League Against Rheumatism (EULAR) recommendations for the management of psoriatic arthritis with pharmacological therapies: 2015 update. Ann Rheum Dis. 2016; 75: 499510.

39. Schoels MM, Aletaha D, Alasti A, Smolen JS. Disease Activity in psoriatic arthritis (PsA): defining remission and treatment success using the DAPSA score. Ann Rheum Dis. 2015; $0: 1-8$. 